\title{
Compositional dependence of infrared absorption spectra of crystalline silicate
}

\section{Natural and synthetic olivines}

\author{
C. Koike ${ }^{1}$, H. Chihara ${ }^{1,2}$, A. Tsuchiyama ${ }^{2}$, H. Suto ${ }^{3}$, H. Sogawa $^{1}$, and H. Okuda ${ }^{4}$ \\ 1 Kyoto Pharmaceutical University, Yamashina, Kyoto 607-8412, Japan \\ 2 Department of Earth and Space Science, Osaka University, Toyonaka, Osaka 560-0043, Japan \\ 3 Subaru Telescope, National Astronomical Observatory of Japan, Hilo, Hawaii, USA \\ ${ }^{4}$ Gunma Astronomical Observatory, Agatsuma, Gunma 377-0702, Japan
}

Received 30 July 2002 / Accepted 10 December 2002

\begin{abstract}
The mass absorption spectra of crystalline olivine particles with different $\mathrm{Mg} /(\mathrm{Mg}+\mathrm{Fe})$ ratios, between forsterite $\left(\mathrm{Mg}_{2} \mathrm{SiO}_{4}, \mathrm{Fo}\right)$ and fayalite $\left(\mathrm{Fe}_{2} \mathrm{SiO}_{4}, \mathrm{Fa}\right)$, were measured for synthetic and natural samples in the mid- and far-infrared regions. The main strong peaks show a systematic shift to longer wavelengths as the $\mathrm{Mg} /(\mathrm{Mg}+\mathrm{Fe})$ ratio decreases. In the $10-17 \mu \mathrm{m}$ region, these trends are very clear, and the peak positions are a good indicator of the composition of dust grains. In the 20-100 $\mu \mathrm{m}$ region, the trends are also rather clear, though they are slightly complicated; the intensity and width of the various bands become weak and broad, respectively, or the bands disappear as the concentration of Fo decreases. However, the trends are clear only in a limited composition range near Fo and Fa; the peak positions of 30, 50, and $69 \mu \mathrm{m}$ bands of forsterite shift linearly as the Fo concentration decreases to about $\mathrm{Fo}_{70}$. Those of the double band in the $50 \mu \mathrm{m}$ region for Fe-rich olivine shift linearly as the Fa concentration increases from $\mathrm{Fo}_{20}$ to $\mathrm{Fa}$. When the spectral signature of Mg-rich or Fe-rich olivine can be observed, the peak positions in the $20-100 \mu \mathrm{m}$ region are a good indicator of the composition of dust grains.
\end{abstract}

Key words. methods: laboratory - interplanetary medium - star: circumstellar matter - ISM: dust, extinction ISM: lines and bands - infrared: ISM

\section{Introduction}

In addition to the characteristic 11.2-11.3 $\mu \mathrm{m}$ band of olivine in many comets such as Halley, Bradfield, Levy, and Muller (Hanner et al. 1994), many characteristic features in the far infrared region were observed around the circumstellar environment of both evolved and young stars and the Hale-Bopp comet. The majority of these features were identified with Mg-rich crystalline olivine and pyroxene (Waters et al. 1998; Molster et al. 1999; Crovisier et al. 1997; Malfait et al. 1998; Wooden et al. 1999; Molster et al. 2002a,b). Further, the detected $69 \mu \mathrm{m}$ feature in the ISO spectra led to the conclusion that the crystalline olivine is also very Mg-rich comparing with laboratory data dependent on the composition and temperature (Molster et al. 2002c). However, no evidence of the presence of iron-rich silicates has been obtained to date. The absence of Fe-rich silicates is very curious as the most abundant elements forming refractory compounds are $\mathrm{Mg}, \mathrm{Si}$, and $\mathrm{Fe}$ with oxygen. A model of the evolution of mineral dust grains in the circumstellar environment has been discussed; at low temperature

Send offprint requests to: C. Koike, e-mail: koike@mb.kyoto-phu.ac.jp after condensation of forsterite and enstatite, gaseous Fe reacts with Mg-rich silicates and amorphous Fe-rich olivines may condensate (Tielens et al. 1998), or the olivine grains develop a core mantle structure with an Mg-rich olivine in the core and Fe-rich olivine in the mantle, and the remaining fraction of the iron forms metallic iron grains (Gail \& Sedlemeyer 1999). The spectra of crystalline silicates dependent on the $\mathrm{Mg} /(\mathrm{Mg}+\mathrm{Fe})$ ratio is a key to understanding the structure, origin, and evolution of circumstellar and interstellar dust, and possibly to identifying the chemical condensation path of circumstellar minerals. Once the carrier of the observed bands has been identified, laboratory spectra should be carefully compared. Generally speaking, the laboratory spectra vary depending on the chemical composition, temperature, and grain shapes of samples. With regard to laboratory data dependence on composition, we already measured the spectra of olivine with different $\mathrm{Mg} /(\mathrm{Mg}+\mathrm{Fe})$ ratios, including $\mathrm{Fo}_{100}, \mathrm{Fo}_{90}, \mathrm{Fo}_{80}, \mathrm{Fo}_{60}$, and $\mathrm{Fo}_{40}$ (Koike et al. 1993). We have clarified that the peak position of the main bands shifts to a longer wavelength as this ratio decreases. This trend was confirmed for olivine and pyroxene by Jäger et al. (1998), using olivine compositions of $\mathrm{Fo}_{100}, \mathrm{Fo}_{94}, \mathrm{Fo}_{55}$, and fayalite. Chihara et al. (2002) (Paper I) 
investigated and confirmed this trend in more detail for $\mathrm{Mg}-\mathrm{Fe}$ pyroxenes with different $\mathrm{Mg} /(\mathrm{Mg}+\mathrm{Fe})$ ratios. In previous investigations of the spectra of olivine (Koike et al. 1993; Jäger et al. 1998), the spectra in the far infrared region were found to change drastically between forsterite and fayalite; a single peak of forsterite at $50 \mu \mathrm{m}$ was changed to double bands of fayalite, and a strong peak of forsterite at $69 \mu \mathrm{m}$ faded away for fayalite. The significance of these detailed trends, especially near Mg-rich and Fe-rich olivine, is not yet clear. For Ferich olivine, only two spectra, that of fayalite and that of $\mathrm{Fo}_{6}$, by Jäger et al. (1998) and Mennela et al. (1998), respectively, have been measured. Further, the samples synthesized by Jäger et al. (1998) are phase-separated (inhomogeneous) materials. It is advantageous to measure the spectra of homogeneous samples so that they can be used as reference materials for spectroscopy. In order to examine in more detail how these peak positions shift with the $\mathrm{Mg} /(\mathrm{Mg}+\mathrm{Fe})$ ratios near forsterite and fayalite, we obtained especially $\mathrm{Mg}$-rich olivine and Fe-rich olivine samples. Following our Paper I, we measured the absorption spectra of the whole range of olivine solid solutions from forsterite to fayalite in the mid-infrared region $(5-25 \mu \mathrm{m})$ and the far-infrared region (longer than $25 \mu \mathrm{m}$ ). Previous data (Koike et al. 1993) in the mid-infrared region had low resolution, and those in 25-35 $\mu \mathrm{m}$ were not smoothly connected with the data in the mid- and the far-infrared region. All these data were re-measured in the present study. We report the spectra of an olivine series, and clarify the correlation between the peak positions and the $\mathrm{Mg} /(\mathrm{Mg}+\mathrm{Fe})$ ratio in detail.

\section{Experiment}

Olivine is a solid solution having as its two end members forsterite $\mathrm{Mg}_{2} \mathrm{SiO}_{4}(\mathrm{Fo})$ and fayalite $\mathrm{Fe}_{2} \mathrm{SiO}_{4}(\mathrm{Fa})$, and is expressed as $\left(\mathrm{Mg}_{x}, \mathrm{Fe}_{1-x}\right)_{2} \mathrm{SiO}_{4}(0 \leq x \leq 1)$. The composition of olivine is expressed as $\mathrm{Fo}_{X}$, where $X$ is the mole percentage of forsterite $(X=100 x)$. In this study, both the synthetic and natural olivines are prepared as listed in Table 1. Synthetic Fo was made by the Czochralski (CZ) method (Takei et al. 1974), and Fa was made by the floating-zone (FZ) method (Takei 1978). The other synthetic olivine samples - Fo80, Fo60, Fo40, Fo20, Fo15, and Fo10 - were produced at $1200{ }^{\circ} \mathrm{C}$ by heating a mixture of $\mathrm{MgO}, \mathrm{SiO}_{2}$, and $\mathrm{FeO}$ powder under controlled $\mathrm{O}_{2}$ fugacity at atmospheric pressure. This method is the same as that mentioned in Paper I for synthesizing pyroxenes. None of the samples showed any phase-separated materials or any inhomogeneity under a SEM, based on the finding that the back scattered electron images were uniform.

The composition of olivine samples was determined by an EPMA (electron probe micro analyzer) at Osaka University (JEOL733). For the EPMA investigations, the chemical composition of natural samples are from $\mathrm{Fo}_{92.1}$ to $\mathrm{Fo}_{77}$ as listed in Table 1. Natural olivines contain minor compounds such as $\mathrm{MnO}, \mathrm{NiO}$, and $\mathrm{CaO}$ (below $0.2 \mathrm{~mol} \%$ ), and the spectral influence of these minor compounds might be negligible. The samples of AK, Fo80, Fo60, and Fo40 samples, which are the same as those in Koike et al. (1993), were very finely ground. The errors of chemical compositions in these samples are rather large compare to those in other samples, due to the fact that the radius of these particles was rather small relative to the beam radius of the electron. The synthetic Fo, Fa and natural samples are single crystal, whereas other synthetic olivines are polycrystal. The synthesized and natural samples were crushed and ground in an agate mortar. After the large particles had been excluded via sedimentation in alcohol, the fine particles were well mixed with $\mathrm{KBr}$ or polyethylene powder in an agate mortar. These fine particles were embedded in $\mathrm{KBr}$ or polyethylene. The diameter of the $\mathrm{KBr}$ pellets was $13 \mathrm{~mm}$. Polyethylene pellets (sheets) were made after heating the mixture, and the diameters of the pellets were about $20-30 \mathrm{~mm}$ according to the total amount of the mixture. The transmittances $T$ of each $\mathrm{KBr}$ and polyethylene pellet were measured from $7000 \mathrm{~cm}^{-1}$ to $50 \mathrm{~cm}^{-1}$. The spectrometers used were the Jasco FT/IR-350 and the Nicolet FTIR (Nexus 670) at a resolution of $1 \mathrm{~cm}^{-1}$. In the far-infrared region $\left(250-50 \mathrm{~cm}^{-1}\right)$, the spectra were also measured, at the lower resolution of $4 \mathrm{~cm}^{-1}$ to avoid interference fringe. The mass absorption coefficient, $\kappa$, is derived as

$$
\kappa=\frac{S}{M} \ln \left(\frac{1}{T}\right),
$$

where $S$ is the surface area of the sample pellet, $M$ is the mass of the sample included in the $\mathrm{KBr}$ or polyethylene pellets, and $T$ is the transmittance of the sample pellet. We ascertained the reproduction of the mass absorption coefficients for each sample, changing the ratio of the sample in $\mathrm{KBr}$ or polyethylene. The absorption spectra by $\mathrm{KBr}$ and polyethylene pellets were smoothly connected, and they overlapped in wide wavelength range from approximately the $17 \mu \mathrm{m}$ to the $25 \mu \mathrm{m}$ region. Typically, the present data shorter than $20 \mu \mathrm{m}$ are from $\mathrm{KBr}$ pellets, and those longer than $20 \mu \mathrm{m}$ are from the polyethylene pellets.

\section{Results}

The spectra of all samples are shown as mass absorption coefficients $\kappa\left(\mathrm{cm}^{2} / \mathrm{g}\right)$ in Fig. 1. The previous data for natural olivine for AK (Ichinomegata Akita, Fo 89.3 $_{\text {) }}$ in the far-infrared region (Koike et al. 1993) are also included. The revised peak positions in the mid-infrared region with high resolution (Koike et al. 1993) are blue shifted by about $0.1 \sim 0.2 \mu \mathrm{m}$ compared with their positions in previous data (Koike et al. 1993).

Peak positions of all samples in $\mu \mathrm{m}$ are listed in Table 2. For all bands, the correlation between the peak positions of each band and the concentration of Fo (mol \%), $X$, is plotted in Fig. 2. Based on Figs. 1 and 2, we examine the peak positions of each band dependent on the chemical composition.

\subsection{Peak shifts as Fa content increases}

The spectra of the synthetic olivine gradually changed from forsterite to fayalite as the $\mathrm{Fe}$ content increased, and this change became distinct in the far-infrared region in particular, as shown in Fig. 1. As for natural olivine, its chemical compositions were limited to a narrow range from $\mathrm{Fo}_{92.1}$ to $\mathrm{FO}_{77}$. The spectra of natural samples were more similar to each other in the mid- and far-infrared regions than to the spectra of synthetic olivine. With regard to the $70 \mu \mathrm{m}$ band, the peak positions of 
Table 1. Natural and synthetic olivine samples.

\begin{tabular}{|c|c|c|c|}
\hline \multirow{2}{*}{$\begin{array}{l}\text { sample } \\
\text { name }\end{array}$} & & \multicolumn{2}{|c|}{ chemical composition $(\mathrm{mol} \%)$} \\
\hline & & concentration of $\mathrm{Fo}$ & minor compounds \\
\hline natural & location & & \\
\hline $\mathrm{KH}$ & Kohistan, Pakistan & $92.1 \pm 0.3$ & $\mathrm{MnO} \leqq 0.1, \mathrm{NiO} \leqq 0.3$ \\
\hline SJ & St. Jones Island, Egypt & $91.4 \pm 0.4 \quad(17)$ & $\mathrm{MnO} \leqq 0.1$ \\
\hline $\mathrm{SC}$ & San Carlos, Arizona, USA & $90.7 \pm 0.2$ & $\mathrm{MnO} \leqq 0.1, \mathrm{NiO} \leqq 0.4$ \\
\hline $\mathrm{AK} *$ & Ichinomegata, Akita, Japan & 89.3 & $\mathrm{NiO} \leqq 0.3$ \\
\hline HW & Black sand beach, Hawaii, USA & $86.5 \pm 0.3$ & $\mathrm{MnO} \leqq 0.2$ \\
\hline MY & Miyake Island, Tokyo, Japan & $84.4 \pm 0.1$ & $\mathrm{MnO} \leqq 0.2, \mathrm{CaO} \leqq 0.2$ \\
\hline NS & Nishigatake, Saga, Japan & $84.2 \pm 0.2 \quad(26)$ & $\mathrm{MnO} \leqq 0.2, \mathrm{CaO} \leqq 0.2$ \\
\hline $\mathrm{NV}$ & Nivitigala, Sri Lanka & (1) & \\
\hline synthetic & synthetic method & & \\
\hline Fo & $\mathrm{CZ}_{\text {method }^{(1)}}$ & - & \\
\hline Fo80* & heating at $1200^{\circ} \mathrm{C}$ & $75.2 \pm 2.4$ & \\
\hline Fo60 * & heating at $1200^{\circ} \mathrm{C}$ & $56.8 \pm 2.2$ & \\
\hline Fo $40 *$ & heating at $1200^{\circ} \mathrm{C}$ & $39.5 \pm 2.1$ & \\
\hline Fo20 & heating at $1200^{\circ} \mathrm{C}$ & $21.8 \pm 0.4$ & \\
\hline Fo15 & heating at $1200^{\circ} \mathrm{C}$ & $15.9 \pm 0.2$ & \\
\hline Fo10 & heating at $1200^{\circ} \mathrm{C}$ & $11.0 \pm 0.2$ & \\
\hline $\mathrm{Fa}$ & FZ method ${ }^{(2)}$ & - & \\
\hline
\end{tabular}

natural olivine were definitely shifted to a longer wavelength as the Fe content increased.

The trend for each band seemed no different between synthetic and natural olivine; the same symbols are shown in Fig. 2. The sizes of symbols indicate the strength of the peaks. From Fig. 2, the following trends have been found.

(1) All peaks are shifted toward longer wavelengths as the Fo content decreases (i.e., the Fa content increases).

(2) For several strong bands, seen at 11.2, 16.4, and $23.7 \mu \mathrm{m}$ of Fo, respectively, peak positions are strongly correlated with the Fo content, $X$. The peak positions in wavenumber $\left(\mathrm{cm}^{-1}\right), v$, are nearly linear with $X$.

\subsection{In the $10-20 \mu m$ region}

The three peaks of forsterite, at 1000,893 , and $614 \mathrm{~cm}^{-1}$ $(10,11.2$, and $16.3 \mu \mathrm{m})$, are strong, and two peaks, at 962 and $840 \mathrm{~cm}^{-1}$ (10.4 and $\left.11.9 \mu \mathrm{m}\right)$, are weak. The peak positions of all these bands shift linearly to longer wavelengths as the $\mathrm{Fa}$ content increases. Forsterite and all natural olivines have a $500 \mathrm{~cm}^{-1}(20 \mu \mathrm{m})$ band with a shoulder at about $550 \mathrm{~cm}^{-1}$ $(18 \mu \mathrm{m})$. For the Fo80 sample, this $20 \mu \mathrm{m}$ band is very broad with a single structure. As the Fa content increases below $\mathrm{Fo}_{60}$, this band broadens and splits into a double structure, and $\mathrm{Fa}$ sample shows clearly double bands at 508 and $478 \mathrm{~cm}^{-1}(19.7$ and $20.9 \mu \mathrm{m})$, respectively.

\subsection{In the $20-30 \mu m$ region}

The strong $422 \mathrm{~cm}^{-1}(23.7 \mu \mathrm{m})$ band (solid square marks in Fig. 2) of forsterite (dotted arrows in Fig. 1) becomes weaker as the Fo content decreases up to the Fo60 sample (including all natural samples). The Fo40 sample shows nearly the same strength as the next band of $345 \mathrm{~cm}^{-1}(29 \mu \mathrm{m})$, whereas below the Fo20 sample this band becomes weaker than the next band of $332 \mathrm{~cm}^{-1}(30 \mu \mathrm{m})$. With regard to the double structure of forsterite at $383 \mathrm{~cm}^{-1}(26.1 \mu \mathrm{m})$ and at $364 \mathrm{~cm}^{-1}$ $(27.5 \mu \mathrm{m})$ (bold arrows in Fig. 1) (full star marks in Fig. 2), natural olivines $(\mathrm{KH}, \mathrm{SJ}, \mathrm{SC}, \mathrm{AK}, \mathrm{HW})$ also show a double structure at about 380 and $360 \mathrm{~cm}^{-1}(26$ and $27 \mu \mathrm{m})$. This double structure becomes a single band and broadens for the Fo80 sample and natural olivine (MY, NS, NV). This broad band becomes strong and shifts to $313 \mathrm{~cm}^{-1}(31.9 \mu \mathrm{m})$ of fayalite as the Fa content increases. The strong band of $313 \mathrm{~cm}^{-1}(31.9 \mu \mathrm{m})$ of fayalite splits into double bands at 26 and $27 \mu \mathrm{m}$ for forsterite.

\subsection{The $33 \mu \mathrm{m}$ band}

The strong band at $296 \mathrm{~cm}^{-1}(33.8 \mu \mathrm{m})$ with two sharp peaks of forsterite becomes a single band for $\mathrm{FO}_{92.1}-\mathrm{FO}_{77}$ composition, and becomes very weak for $\mathrm{Fo}_{56.8}$ and $\mathrm{Fo}_{39.5}$ composition. The band appears weakly for the Fo10 sample, and for Fa the band appears clearly at $257 \mathrm{~cm}^{-1}(38.9 \mu \mathrm{m})$.

\subsection{The $50 \mu \mathrm{m}$ and $70 \mu \mathrm{m}$ bands}

In the far infrared region, a strong peak appears at $200 \mathrm{~cm}^{-1}$ $(50 \mu \mathrm{m})$ and $144 \mathrm{~cm}^{-1}(69.6 \mu \mathrm{m})$ for forsterite, and these peaks are also sensitive to the chemical composition.

With regard to the $50 \mu \mathrm{m}$ band, the peak also appears for five natural olivines $\left(\mathrm{Fo}_{92.1}-\mathrm{Fo}_{86.5}\right)$. In the case of the MY, NS, and NV samples, the $50 \mu \mathrm{m}$ peak appears, but not clearly. 


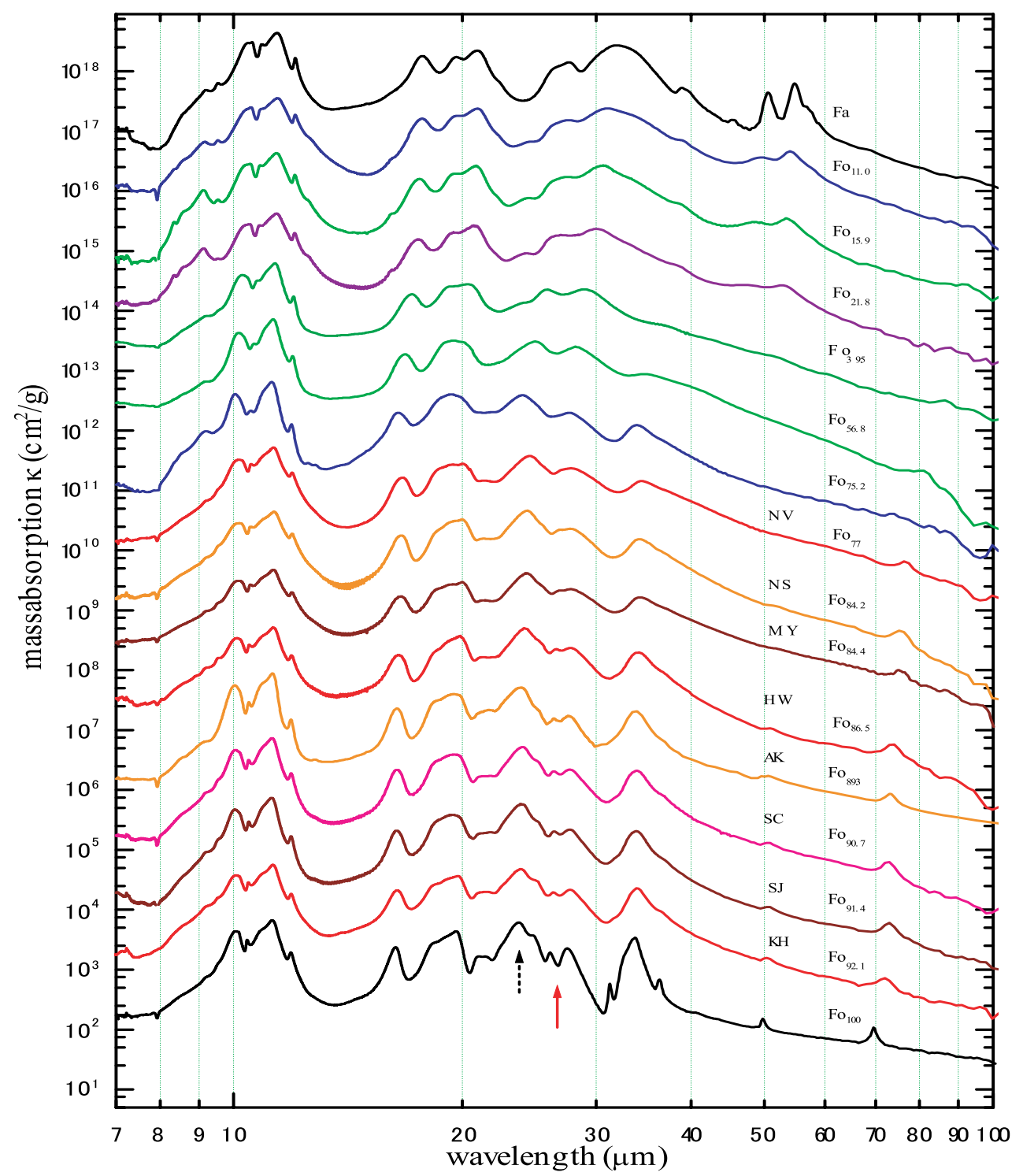

Fig. 1. Spectra of synthetic olivine and natural olivine. Each spectrum, indicating by its composition, is multiplied the under spectrum by 10 . The dotted and bold arrows indicate $23.7 \mu \mathrm{m}$ and the double structure of forsterite, respectively; see Sect. 3.3.

This band does not appear for synthetic olivine (Fo80-Fo40 samples). In the case of the Fo20 sample, very weak double bands appear in the $50 \mu \mathrm{m}$ region. These double bands become clearer and stronger as the Fa content increases, and for Fa sample two very strong bands appear at 198 and at $183 \mathrm{~cm}^{-1}(50.6$ and $54.7 \mu \mathrm{m}$ ).

With regard to the $70 \mu \mathrm{m}$ band, forsterite shows a sharp and distinct band at $144 \mathrm{~cm}^{-1}(69.6 \mu \mathrm{m})$. In the case of natural olivine, the $70 \mu \mathrm{m}$ peak clearly appears, and shifts to $72 \mu \mathrm{m}$ (KH), $73 \mu \mathrm{m}$ (SJ and SC), 73.2 $\mu \mathrm{m}$ (AK), 74.1 $\mu \mathrm{m}$ (HW), $74.9 \mu \mathrm{m}$ (MY), $75.2 \mu \mathrm{m}$ (NS), and $76.3 \mu \mathrm{m}$ (NV). Even in the case of NV, which has a $\mathrm{Fo}_{77}$ composition, the $70 \mu \mathrm{m}$ peak clearly appears at $76.3 \mu \mathrm{m}$. All natural olivines definitely show this band, and this peak position shifts to the longer wavelength as the Fa content increases. However, all the synthetic olivines except forsterite show this band very weakly beyond $70 \mu \mathrm{m}$. The peak position of the fayalite shows much more vaguely at about 103 and $94.3 \mathrm{~cm}^{-1}$ (97 and $106 \mu \mathrm{m}$ ), and is not added to the figure due to large errors, whereas the bulk sample of fayalite shows the bands at 94 and $109 \mu \mathrm{m}$ (Hofmeister et al. 1997). In the far-infrared region, the $\mathrm{SiO}_{4}$ groups in olivines are distorted, partly due to the influence of Fe incorporation. The small iron content significantly reduces the intensity of some peaks, including those at 33,50 , and $70 \mu \mathrm{m}$.

\section{Discussions}

\subsection{Peak shifts depending on bands}

The peak positions for forsterite $\left(\mathrm{Fo}_{100}\right)$, natural olivine $\left(\mathrm{Fo}_{94}, \mathrm{Fo}_{55}\right)$, and fayalite determined by Jäger et al. (1998) are plotted as open circles; those for natural olivine ( $\mathrm{Fo}_{90}$ and $\mathrm{Fo}_{6}$ ) determined by Mennella et al. (1998) as asterisks; and those for forsterite $\left(\mathrm{Fo}_{100}\right)$ and natural olivine $\left(\mathrm{FO}_{89}\right)$ determined by Bowey et al. (2001) as open triangles in Fig. 2. The trends of their data resemble those of ours. Although Jäger et al. (1998) showed forsterite having three bands in the region 


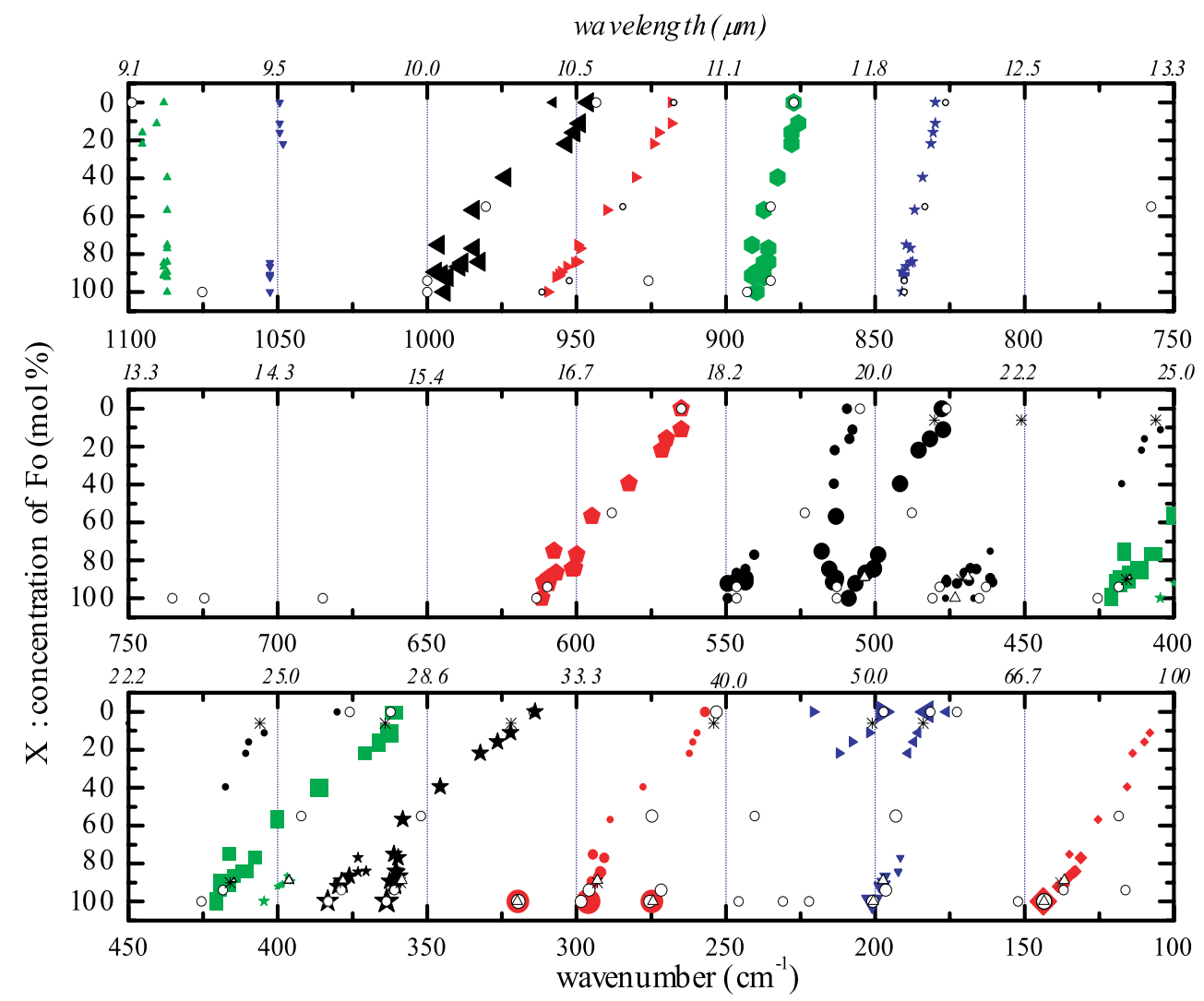

Fig. 2. Peak shifts of absorption bands depending on chemical composition. Solid symbols are synthetic and natural samples. Open circles $\circ$, asterisk $*$, and open triangle $\Delta$ are from Jäger's (1998), Mennela's (1998), and Bowey's data (2001), respectively. The sizes of the symbols indicate the strength of peaks.

between $40-50 \mu \mathrm{m}$, these bands do not appear for our or Bowey's forsterite. Further, Jäger's forsterite has other bands at 13.6, 13.8, 14.6, and $65.7 \mu \mathrm{m}$, and these bands do not appear in our or Bowey's forsterite. This difference may be due to the fact that Jäger's forsterite sample was inhomogeneous and some other compounds (or impurity) remain.

From Fig. 2, there seems generally to be no difference between natural and synthetic olivine. The peak positions of each band in wavenumber $\left(\mathrm{cm}^{-1}\right), v$, linearly shift as the Fo content, $X$, decreases; that is, $v$ are nearly in proportion to $X$, although some change appears around chemical composition $\mathrm{Fo}_{80}-\mathrm{FO}_{70}$. The linear fitting equations for the respective bands are listed in Table 3 . The proportional coefficients or the slopes between $v$ and $X$ for each band ( $v$ is proportional to $X$ ) that are shown in Fig. 2 and Table 3 evidently differ. For example, the slopes are 0.15 for 11.2 micron band, 0.12 for 12 micron band, 0.51 for 16 micron band, 0.65 for 23.7 micron band, and 0.37 for 70 micron band. These results are different from those determined by Jäger et al. (1998), in which the shifts in wavenumber are constant for the different bands and proportional to the [FeO] content (their slope is 0.56). From Fig. 2 and Table 3, it can be seen that the peak shifts or the slopes are very small for 11.2 and 11.9 micron bands of Fo. For other bands, the peak shifts are very clear, and their slopes are about 0.4-0.7. With regard to the 26.1 and 27.5 micron bands, these double bands become single in the Fo80 sample. Including the medium 26.1 micron band instead of the strong 27.5 micron band for
$X$ regions from 100 to 80 , the fitting slope becomes 0.68 , which is similar to that of the 23.7 micron band.

The positions in the $10-17 \mu \mathrm{m}$ region are good indicators of the $\mathrm{Mg} /(\mathrm{Mg}+\mathrm{Fe})$ ratio. However, in the $20-100 \mu \mathrm{m}$ region, the peak shifts are rather complicated, due to the fact that these bands become weak, broad, or undistinguished as the Fa content increases. Lastly, we discussed the differences in spectra of the $70 \mu \mathrm{m}$ band between natural and synthetic olivine. All natural olivine samples $\left(\mathrm{Fo}_{92.1}-\mathrm{Fo}_{77}\right)$ clearly showed a $70 \mu \mathrm{m}$ band similar to forsterite. Further, according to Jäger et al. (1998), natural hortonolite $\left(\mathrm{Fo}_{55}\right)$ shows a weak peak at $84.4 \mu \mathrm{m}$. In contrast, synthetic Fo80 and Fo60 samples show a very weak band at 74.1 and $79.8 \mu \mathrm{m}$, respectively. These findings might explain differences in crystal state (single crystal or polycrystal), or the preferential concentration of $\mathrm{Mg}$ and Fe elements to the M1- or M2-site (Aikawa et al. 1985; Morimoto 1993), but more information is needed and additional measurements of synthetic olivine must be examined.

\subsection{The chemical route of silicate formation}

Observation by ISO shows that ubiquitous $\mathrm{Mg}$-rich crystalline silicates (forsterite and enstatite) exist in the circumstellar region. Thus far, crystalline fayalite or Fe-rich silicates has not detected by ISO observation. As mentioned in the Introduction, evolution models to explain the absence of Fe-rich silicates in the circumstellar environments have been suggested by 
Table 2. Peak positions in $\mu \mathrm{m}$ at room temperature. The first and second lines are the sample name and concentration of Fo (mol \%), respectively. $*$ : very weak peak.

\begin{tabular}{|c|c|c|c|c|c|c|c|c|c|c|c|c|c|c|c|}
\hline Fo & $\mathrm{KH}$ & SJ & $\mathrm{SC}$ & $\mathrm{AK}$ & HW & MY & NS & $\mathrm{NV}$ & Fo80 & Fo60 & Fo40 & Fo20 & Fo15 & Fo10 & $\mathrm{Fa}$ \\
\hline 100 & 92.1 & 91.4 & 90.7 & 89.3 & 86.5 & 84.4 & 84.2 & 77 & 75.2 & 56.8 & 39.5 & 21.8 & 15.9 & 11.0 & 0 \\
\hline $9.2 *$ & $9.2 *$ & $9.2^{*}$ & $9.2^{*}$ & $9.2^{*}$ & $9.2^{*}$ & $9.2^{*}$ & $9.2^{*}$ & $9.2^{*}$ & 9.2 & $9.2 *$ & $9.2^{*}$ & 9.1 & 9.1 & 9.2 & 9.2 \\
\hline \multirow[t]{2}{*}{$9.5^{*}$} & $9.5^{*}$ & $9.5^{*}$ & $9.5^{*}$ & & $9.5^{*}$ & $9.5^{*}$ & & & & & & $9.5^{*}$ & $9.5^{*}$ & 9.5 & 9.5 \\
\hline & & & & & & & & & & & & & & & $10.4^{*}$ \\
\hline 10.06 & 10.16 & 10.05 & 10.05 & 10.03 & 10.11 & 10.12 & 10.18 & 10.16 & 10.04 & 10.16 & 10.27 & 10.49 & 10.52 & 10.54 & 10.57 \\
\hline 10.42 & 10.46 & 10.46 & 10.46 & 10.47 & 10.49 & 10.52 & 10.53 & 10.54 & 10.53 & 10.64 & 10.75 & 10.82 & 10.84 & 10.89 & 10.89 \\
\hline 11.24 & 11.28 & 11.22 & 11.23 & 11.24 & 11.27 & 11.27 & 11.29 & 11.29 & 11.22 & 11.27 & 11.33 & 11.39 & 11.39 & 11.42 & 11.40 \\
\hline 11.9 & 11.9 & 11.9 & 11.9 & 11.9 & 11.9 & 11.9 & 11.9 & 11.9 & 11.9 & 12.0 & 12.0 & 12.0 & 12.0 & 12.1 & 12.1 \\
\hline \multirow[t]{2}{*}{16.4} & 16.5 & 16.4 & 16.4 & 16.4 & 16.5 & 16.6 & 16.7 & 16.7 & 16.5 & 16.8 & 17.2 & 17.5 & 17.6 & 17.7 & 17.7 \\
\hline & & & & & & $18.4^{*}$ & & $18.5^{*}$ & & & & & & & \\
\hline $18.2^{*}$ & $18.3^{*}$ & $18.4^{*}$ & $18.3^{*}$ & $18.4^{*}$ & $18.3^{*}$ & $19.4^{*}$ & & & & & $19.5^{*}$ & 19.5 & 19.7 & 19.7 & 19.6 \\
\hline 19.7 & 19.8 & 19.5 & 19.5 & 19.5 & 19.9 & 20.0 & 20.0 & 20.0 & 19.3 & 19.5 & 20.3 & 20.6 & 20.8 & 21.0 & 20.9 \\
\hline 20.8 & $21.2^{*}$ & $21 *$ & $21^{*}$ & $21^{*}$ & & & & & & & & & & & \\
\hline $21.4^{*}$ & & $21.7^{*}$ & $21.3^{*}$ & $21.7^{*}$ & $21.3^{*}$ & $21.5^{*}$ & $21.4^{*}$ & $21.5^{*}$ & $21.7^{*}$ & & 24. $0^{*}$ & $24.3^{*}$ & $24.4^{*}$ & $24.7^{*}$ & $26.3^{*}$ \\
\hline 23.7 & 24.0 & 23.9 & 24.0 & 23.9 & 24.1 & 24.3 & 24.3 & 24.6 & 24.0 & 25.0 & 25.9 & 27.0 & 27.3 & 27.6 & 27.7 \\
\hline 24.8 & & 25.1 & 25.1 & 25.3 & 25.2 & & & & & & & & & & \\
\hline 26.1 & 26.3 & 26.4 & 26.4 & 26.4 & 26.6 & $26.8^{*}$ & $27.0^{*}$ & $26.8^{*}$ & & & & & & & \\
\hline 27.5 & 27.7 & 27.7 & 27.7 & 27.6 & 27.8 & 27.8 & 27.7 & 27.8 & 27.7 & 27.9 & 28.9 & 30.1 & 30.6 & 31.1 & 31.9 \\
\hline \multicolumn{16}{|l|}{31.3} \\
\hline 33.8 & 33.9 & 33.9 & 34.0 & 33.9 & 34.2 & 34.3 & 34.2 & 34.4 & 34.0 & 34.6 & 36 & $38.1^{*}$ & $38.3^{*}$ & $38.5^{*}$ & $38.9 *$ \\
\hline \multicolumn{16}{|l|}{36.4} \\
\hline & & & & & & & & & & & & & & & 45.3 \\
\hline & & & & & & & & & & & & $47.1^{*}$ & 48.1 & 49.5 & 50.6 \\
\hline \multirow[t]{3}{*}{49.7} & 50.3 & 50.3 & 50.8 & 50.6 & 50.8 & $52 *$ & $52 *$ & $(52 ?)$ & & & & & & & \\
\hline & & & & & & & & & & & & $52.9^{*}$ & 53.5 & 53.9 & 54.7 \\
\hline & & & & & & & & & & & & & & & $56.8^{*}$ \\
\hline \multirow[t]{2}{*}{69.6} & 72.0 & 73.0 & 73.0 & 73.2 & 74.1 & 74.9 & 75.2 & 76.3 & $74.1^{*}$ & $79.8^{*}$ & $86.4^{*}$ & $87.9^{*}$ & $91.0^{*}$ & $92.6^{*}$ & $97 * ?$ \\
\hline & & & & & & & & & & & & & & & $105.8 * ?$ \\
\hline
\end{tabular}

Tielens et al. (1998) and by Gail \& Sedlmayr (1999). According to the model by Tielens et al. (1998), if Fe-rich olivine in the circumstellar region crystallizes in response to some annealing mechanism, detection of strong double peaks at 50.6 and $54.8 \mu \mathrm{m}$ can be expected. According to the model by Gail \& Sedlmayr (1999), the average composition $x$ of olivine grains is about $0.7-0.6$, and the surface composition of olivine $x$ is about $0.4-0.7$ as dependent on the magnitude of the ion exchange coefficient. In the case in which $x$ is 4070 mole \% (i.e., when $\mathrm{Fo}_{40}-\mathrm{Fo}_{70}$ ), there is no feature or there is a very weak peak in the $50 \mu \mathrm{m}$ and $70 \mu \mathrm{m}$ regions, respectively, in the present olivine spectra. Determining the spectral signatures of Fe-rich olivine could be resolved this problem. The spectral signatures appear at $50 \mu \mathrm{m}$, where there is a strong single peak of forsterite or a double peak of fayalite, and at the $70 \mu \mathrm{m}$ band, where the peak position shifts to a longer wavelength as the Fo content decreases.

In the case of the $70 \mu \mathrm{m}$ band, the intensity of natural olivine is weak compared to that of forsterite. Based on the ISO observations of evolved stars, the detected $70 \mu \mathrm{m}$ band was around $69 \mu \mathrm{m}$, and was identified with forsterite, taking into account the temperature dependence (Molster et al. 2002c; Bowey et al. 2001). The peak position of present natural olivine does not shift to below $70 \mu \mathrm{m}$, even after cooling to $4 \mathrm{~K}$ from the peak shift of natural olivine $\mathrm{Fo}_{90}$ at $20 \mathrm{~K}$ (Mennela et al. 1998). As mentioned above, the intensity and peak position of this $70 \mu \mathrm{m}$ band are very sensitive to chemical composition. It is very interesting how the intensity and peak position of the band changed for the limited composition Fo-Fo ${ }_{90}$. Not only the $70 \mu \mathrm{m}$ band but also the 33 and $50 \mu \mathrm{m}$ bands were sensitive to chemical composition and were largely changed near Fo and Fa. The more detailed trends of these bands are interesting, and further investigation of the limited compositions of $\mathrm{Fo}-\mathrm{Fo}_{90}$ and $\mathrm{Fo}_{10}-\mathrm{Fa}$ are needed.

\subsection{Peak shifts of olivine and pyroxene}

For olivine samples, the peak positions in the mid-infrared region shift linearly as Fo concentration decreases, whereas in the far-infrared region this trend appears clearly only near the end members; the peak positions of 33,50 , and $69 \mu \mathrm{m}$ bands of Fo clearly shift linearly from $\mathrm{Fo}$ to about $\mathrm{Fo}_{70}$, and the peak positions of Fa at $50 \mu \mathrm{m}$ bands clearly shift linearly as Fa concentartion decreases to about $\mathrm{Fo}_{20}$. In contrast, these trends appear slightly different for pyroxene (Paper I). The compositions of pyroxene are limited to En-En50 and Fs, and the peak shifts 
Table 3. The correlation between peak position $v$ in wavenumber $\left(\mathrm{cm}^{-1}\right)$ and Fo content, $X$.

\begin{tabular}{lc}
\hline \hline peak band of Fo & linear fitting equation \\
\hline $10 \mu \mathrm{m}$ & $v=0.53 X+946$ \\
$10.4 \mu \mathrm{m}$ & $v=0.44 X+915$ \\
$11.2 \mu \mathrm{m}$ & $v=0.15 X+876$ \\
$11.9 \mu \mathrm{m}$ & $v=0.12 X+829$ \\
$16.3 \mu \mathrm{m}$ & $v=0.51 X+562$ \\
$23.7 \mu \mathrm{m}$ & $v=0.65 X+359$ \\
$27.5 \mu \mathrm{m}$ & $v=0.48 X+320$ \\
$27.5 \mu \mathrm{m} *$ & $v=0.68 X+316$ \\
$33.4 \mu \mathrm{m}$ & $v=0.43 X+257$ \\
$69 \mu \mathrm{m}$ & $v=0.37 X+104$ \\
$50 \mu \mathrm{m}(\mathrm{Fo})$ & $v=0.48 X+154$ \\
$50 \mu \mathrm{m}(\mathrm{Fa})$ & $v=0.67 X+197$ \\
$50 \mu \mathrm{m}(\mathrm{Fa})$ & $v=0.29 X+183$ \\
\hline
\end{tabular}

Here, as was the case for $27.5 \mu \mathrm{m}^{*}$, peaks are fitted including the $26.1 \mu \mathrm{m}$ band instead of the $27.5 \mu \mathrm{m}$ band in the range of $X$ from 100 to 80 . For $50 \mu \mathrm{m}(\mathrm{Fo})$ and $(\mathrm{Fa})$, peaks are fitted for the $50 \mu \mathrm{m}$ bands only for a limited range of $X$ in the case of Fo-rich and Fa-rich olivine, respectively.

in the mid-infrared region do not appear as clearly as those of olivine, whereas the peak shifts in the far-infrared region, especially at around the 40-50 and $70 \mu \mathrm{m}$ regions, clearly appear. Up to now, crystalline olivine and pyroxene had been detected by ISO observation. This trend in peak shifts of olivine and pyroxene will contribute to identification of the bands and clarify overall chemical composition. In particular, from the peak shifts of olivine in the mid-infrared region and those of pyroxene in the far-infrared region, we can easily determine the chemical composition of crystalline silicates. Spectra with high resolution around the circumstellar environment will provide a clue to clarify the growth of circumstellar dust.
Acknowledgements. We thank Professor H. Takei of Osaka University for preparing Fayalite crystal. This work was supported by a Grant-inAid from the Japanese Ministry of Education, Science, and Culture (No. 12440054), and a Research fellowship from the Japan Society for the Promotion of Science for Young Scientists (to Chihara). We thank the referee Dr. J. E. Bowey for constructive comments that have improved this paper. We are grateful to Dr. Catherine Ishida for improving expressions of our manuscript.

\section{References}

Aikawa, N., Kumazawa, M., \& Tokonami, M. 1985, Phys. Chem. Minerals, 12, 1

Bowey, J. E., Lee, C., Tucker, C., et al. 2001, MNRAS, 325, 886

Chihara, H., Koike, C., Tsuchiyama, A., Tachibana, S., \& Sakamoto, D. 2002, A\&A, 391, 267 (Paper I)

Crovisier, J., Leech, K., \& Bockelee-Morvan, D., et al. 1997, Science 275, 1904

Gail, H.-P., \& Sedlmayr, E. 1999, A\&A, 347, 594

Hanner, M. S., Lynch, D. K., \& Russell, R. W. 1994, ApJ, 425, 274

Hofmeister, A. M. 1997, Phys. Chem. Minerals, 24, 535

Jäger, C., Molster, F. J., Dorschner, J., et al. 1998, A\&A, 339, 904

Koike, C., Shibai, H., \& Tsuchiyama, A. 1993, MNRAS, 264, 654

Malfait, K., Waelkens, C., Waters, L. B. F. M., et al. 1998, A\&A, 332, L25

Mennella, V., Brucato, J. R., Collangeli, L., et al. 1998, ApJ, 496, 1058

Molster, F. J., Waters, L. B. F. M., Trams, N., et al. 1999, A\&A, 350, 163

Molster, F. J., Waters, L. B. F. M., Tielens, A. G. G. M., et al. 2002a, A\&A, 382, 184

Molster, F. J., Waters, L. B. F. M., \& Tielens, A. G. G. M. 2002b, A\&A, 382, 222

Molster, F. J., Waters, L. B. F. M., Tielens, A. G. G. M., et al. 2002c, A\&A, 382, 241

Morimoto, N. 1993, Rock-forming minerals (Tokyo University press) (in Japanese)

Takei, H. 1978, J. Crystal Growth, 43, 463

Takei, H., Miura, T., \& Morioka, M. 1974, J. Cryst. Growth, 23, 121

Tielens, A. G. G. M., Waters, L. B. F. M., Molster, F. J., \& Justtanont, K. 1998, Ap\&SS, 255, 415

Waters, L. B. F. M., Beintema, D. A., Zijlstra, A. A., et al. 1998, A\&A, 331, L61

Wooden, D. H., Harker, D. E., Woodward, C. E., et al. 1999, ApJ, 517, 1034 